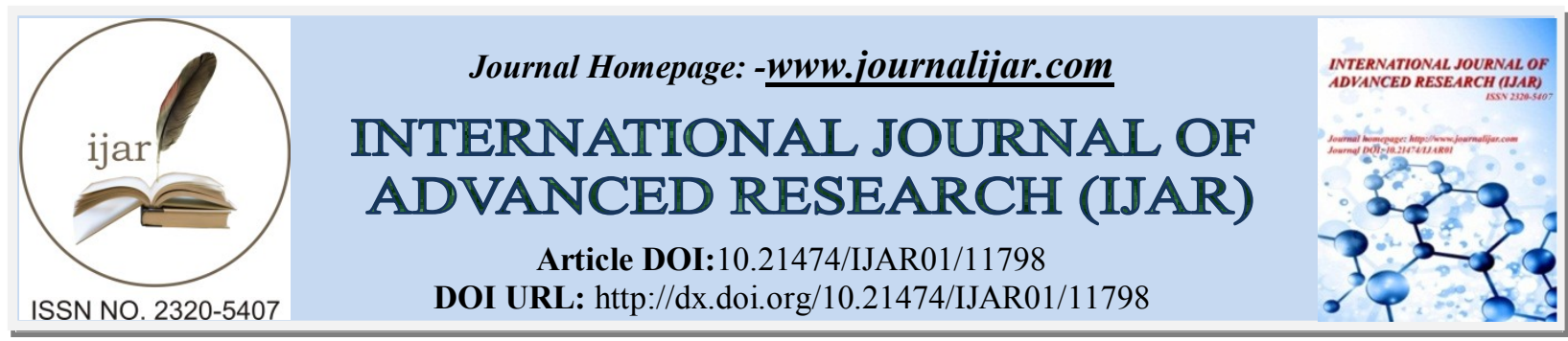

RESEARCH ARTICLE

\title{
SURGICAL AND MEDICAL MANAGEMENT OF SEVER PID WITH PERITUBAL ADHESOLYSIS AND UTERINE LAPAROSCOPIC MYOMECTOMY AS SUCCESSFUL MANAGEMENT OF SECONDARY INFERTILITY
}

\author{
Dr. Ibtessam Saad Hassanin ${ }^{1}$ and Dr. Azhar Schett ${ }^{2}$ \\ 1. MBBCH-Tanta University, Egypt, MD-Ain Shams University, Egypt, Gyne Specialist Prime Hospital, \\ Dubai,UAE. \\ 2. MBCHB-HIGH DIPLOMA OG.-GBOG, Consultant Obs\&gynecology. HOD of Prime Hospital Gyn \\ Department, Dubai,UAE.
}

\section{Manuscript Info}

Manuscript History

Received: 26 July 2020

Final Accepted: 31 August 2020

Published: September 2020

\begin{abstract}
Adhesions are abnormal peritoneal fibrotic bands with varying degrees of stiffness that connect two surfaces that are normally separated in the peritoneal cavity. They are responsible for locating the inflammatory reaction when there is an infection or foreign body carrying oxygen to ischemic tissue [1]. Many serious complications may be associated to adhesions such as chronic pelvic pain and small bowel obstruction and are associated to dyspareunia and secondary infertility [2]. Adhesion formation occurs in $90 \%$ of abdominal and pelvic surgeries, with a lower incidence when the surgery is performed laparoscopically Laparoscopic myomectomy offers a real benefit to infertile patients with uterine fibroids and peritoneal adhesions. The procedure requires a skilled surgeon and laparoscopy technique to minimize adhesion formation and other proven benefits
\end{abstract}

Copy Right, IJAR, 2020,. All rights reserved.

\section{Introduction:-}

Pelvic inflammatory disease (PID) is defined as an inflammation of the upper genital tract due to an infection in women. The disease affects the uterus, fallopian tubes, and/or ovaries. It is typically an ascending infection, spreading from the lower genital tract. The majority of cases PID are related to a sexually transmitted infection. The diagnosis of PID is primarily clinical and should be suspected in female patients with lower abdominal or pelvic pain and genital tract tenderness. During the patient's evaluation, other etiologies of pain including ectopic pregnancy should be considered and ruled out. PID is treated with antibiotics to cover the primary pathogens including Neisseria gonorrhea and Chlamydia trachomatis. Short-term complications include tubo-ovarian or pelvic abscess. Long-term complications include ectopic pregnancy, infertility, and chronic pelvic pain. Early diagnosis and treatment can potentially prevent complications.[1][2][3

Infection of the upper female genital tract leads to inflammatory damage, resulting in scarring, adhesions, and partial or total obstruction of the fallopian tubes. This can result in loss of the ciliated epithelial cells along the fallopian tube lining, resulting in impaired ovum transport and increased risk for infertility and ectopic pregnancy. Additionally, adhesions can lead to chronic pelvic pain.[7]

Women with PID may present with lower abdominal or pelvic pain, vaginal discharge, dyspareunia, 


\section{Complications of PID:}

Delayed treatment of PID has a strong association with worsened outcomes and long-term complications. However, even with timely treatment, long-term complications can occur. One study estimated that for females with PID between 20 to 24 years of age, $18 \%$ would eventually develop chronic pain, $8.5 \%$ would develop ectopic pregnancies, and $16.8 \%$ would struggle with infertility.[13][14][15]

Chronic pelvic pain is seen in as many as one-third of women with PID. The pain is thought to be related to inflammation, scarring, and adhesions from the infectious process. The strongest predictor of developing chronic pelvic pain related to PID is recurrent PID.

Infertility can also result from PID, regardless if the patient is symptomatic or asymptomatic from the pelvic infection. The infection can cause severe damage to the fallopian tubes, including loss of the ciliary epithelial cells of the fallopian tube and occlusion of the tube. The effects on fertility can be pronounced, with some studiesindicating a 5-fold increase in infertility in women with a history of PID. Infertility related to PID is more likely to occur if chlamydia is the infectious cause, if there is a delay in treatment for PID, if the patient has recurrent episodes of PID, or if the case of PID is more severe.

The increased risk for ectopic pregnancy following PID is also related to damage to the fallopian tubes. In one study the rate of ectopic pregnancy following PID is approximately $7.8 \%$ according to one study, while the non-PID ectopic rate is $1.3 \%$.

Patients with PID may develop chronic pain, an ectopic pregnancy, or infertility. About $25 \%$ will have chronic pelvic pain, usually due to adhesions. Impaired fertility affects $10-50 \%$ of females and is usually due to scarring and adhesions within the fallopian tubes. The rates of infertility usually increase with the number of infections episodes. The final problem is an ectopic pregnancy which may occur in $15-60 \%$ of women and is usually due to damage to the fallopian tubes. Some women may develop the Fitz Hugh Curtis Syndrome. Each year there are over 150,000 admissions related to PID. The complication rates in parts of Africa, Asia, and South America where health care is not readily accessible are very high. There are also reports that PID may be associated with a higher risk of stroke and ovarian cancer. [18][19][20] (Level III)

\section{Fibroids and Infertility:}

Uterine fibroids, also known as myomas, are benign growths of the uterus' smooth muscle tissue. They are the most common tumours to be found in the lower abdomen in pre-menopausal women. Fibroids can be found in $30-40 \%$ of all women between the age of 30 and 40 but can occur at any age. Furthermore, they are more prevalent in African women than in women of other ethnicities. The relationship between fibroids and infertility is especially difficult because of the heterogeneity of the fibroids regarding their size, location, and number, as well as the heterogeneity in observed patient populations. Even though a number of studies have attempted to clarify the influence of fibroids on fertility, there have been various, sometimes contradictory, findings and a lack of well-designed trials.

\section{Types of Fibroids:}

Fibroids vary in their size, number, and location. There are three main types of fibroids depending on their location in the uterus. There are many classifications of fibroids.8,9 However, the International Federation of Gynecology and Obstetrics (FIGO) classification is the most recent and it is used worldwide.10 Intramural fibroids (FIGO L3L4) are the most common type in which the fibroid is located in the muscle wall of the womb. Subserosal fibroids (FIGO L5-L7) are situated outside the muscle wall and grow into the direction of the pelvis.

Sub mucosal fibroids (FIGO L0-L2) develop in the myometrium right beneath the endometrium and can grow into the uterine cavity. Furthermore, fibroids can be either pedunculated or directly attached to the uterus. Depending on their location, the symptoms caused by fibroids can vary.

\section{Infertility:}

Infertility is generally defined as the failure to conceive after $\geq 1$ year of unprotected sexual intercourse. There is prevalence of $16 \%$ after 1 year in the general population and a prevalence of $8 \%$ after 2 years. Primary infertility refers to a couple who have had no previous pregnancies before, whereas secondary infertility refers to having failed to conceive following the last pregnancy. Medical investigation is generally recommended after unsuccessful conception after 1 year.14 Male and female factors can lead to infertility, thus both the female and male partner need 
to be investigated. Male factors can include abnormal semen analysis, surgical pelvic procedures, endocrinological conditions, drug abuse, and genetic disorders. Female risk factors for infertility or reduced fertility can be an age of $>35$ years, menstrual irregularity, history of pelvic inflammatory disease or sexually transmitted diseases, endometriosis, over and underweight, previous pelvic surgeries, and the presence of fibroids. 15 Because infertility tends to be multicausal, fibroids (as the sole identified factor for infertility) were only identified in about $2.4 \%$ of patients.

\section{Potential Influence of Fibroids on Fertility from a Biological Perspective:}

There are a number of different mechanisms through which fibroids negatively influence fertility. Firstly, enlarged size or specific location fibroids can hinder the transport of the sperm and egg, as well as their implantation.11,17,18 Secondly, the presence of sub mucosal fibroids seems to influence levels of IL-10 and glycodelin. These cytokines are supposed to support the implantation and early embryonic development. In the presence of fibroids, they appear to be reduced.17 Thirdly, Purohit et al.17 and Yoshino et al.19 have found that fibroids seem to alter uterine contractions leading to an inflammatory reaction in the uterus. The latter may hinder implantation. Fourthly, it is reported that the presence of fibroids leads to a change in the endomyometrial junctional zone. This zone regularly consists of macrophages and natural killer cells that contribute to the endometrial decasualization during implantation. Some studies have shown that, fibroids are associated with the reduction of those cells.20,21 The thickness of fibroid pseudo capsule (a neurovascular bundle surrounding the fibroid that is rich in neurofibres) is considerably higher near the endometrial cavity compared to intramural and sub serous fibroid, suggesting a potential role in fertility.22 Reduced fertility can have a number of causes, and the ones mentioned above are not to be considered as separate or monocausal. The relationship between fibroids and infertility is especially difficult because of the heterogeneity of the fibroids regarding their size, location, and number, as well as the heterogeneity in observed patient populations.21 Even though a number of studies have attempted to clarify the influence of fibroids on fertility, there have been various, sometimes contradictory, findings and a lack of well-designed trials.17 The findings from recent studies and reviews will now be discussed to try to give a recent view on the relationship between fibroids and fertility.

\section{Influence of Fibroids on Fertility:}

The probability of clinical pregnancy, implantation, and live birth in women with fibroids was significantly lower, while abortion rates were higher in comparison to the control group independent of the fibroids' location.21,23 Looking more closely at the fibroids' location, it has been found that intramural fibroids and submucosal fibroids with intracavitary distortion were associated with lower pregnancy, implantation, and live birth rates in comparison with women with no fibroids, whereas there was no difference found for subserosal fibroids.21,24 Generally, submucosal fibroids are associated with a 70\% reduction in delivery rate, intramural with a reduction of 30\%.25-27 It is thought that the size of the fibroid $(2-6 \mathrm{~cm})$ does not impact fertility outcomes; 22 however, recent studies have shown that fibroids size in-fact does have an effect: intramural fibroids of size $>4 \mathrm{~cm}$ were seen to be associated with statistically lower pregnancy rates in comparison to smaller intramural fibroids.28-30 The effect of size could thus be seen in combination with the fibroids' location. In general, one can state that the diverse findings show the need for more controlled, high-quality studies

\section{Treatment options for fibroid and infertility:}

\section{Myomectomy:}

Myomectomy as a potential surgical treatment for fibroids has been explored in many case studies. In general, approximately $50 \%$ of women with infertility and fibroids become pregnant after myomectomy, 16 although the numbers differ depending on the study. Whether performed hysteroscopically or by laparotomy, myomectomy has led to a rise in pregnancy rates; 17,27 however, only the rise regarding submucosal fibroids was statistically significant.17 Then again, the literature review has shown that myomectomy before IVF has significantly increased the success rate in fibroids without submucosal component.28 Because of the divided findings in literature, Purohit et al.17 conclude that the benefit of myomectomy highly depends on the location and size of the fibroids. There is little evidence for a beneficiary effect regarding subserosal fibroids since they appear to have little effect on a woman's fertility. For submucosal fibroids, a positive effect on pregnancy and live birth rates is stated. For intramural fibroids, the evidence remains unclear as there is a higher risk of postoperative complications, such as adhesions that can lead to infertility by themselves.17 A study performed by Casini et al.29 suggested that a pregnancy rate of $56.5 \%$ in women with intramural fibroids and myomectomy in comparison to $41.0 \%$ without myomectomy. However, the higher rate was not statistically significant, thus myomectomy for intramural. 
fibroids should be considered individually depending on exact size, number, and location. 17 Hysteroscopic myomectomy is recommended for fibroids located in the cavity (FIGO L0-L1). In L2, up to $5 \mathrm{~cm}$ fibroids hysteroscopy is possible, however, it might have to be carried out in multiple-stage procedures. It is highly necessary to take into consideration the possible operation related intrauterine adhesions and endometrial damage which are negatively correlated to reproductive outcomes.17 In larger fibroids (FIGO L2 $>5 \mathrm{~cm}$ and above) management by laparotomy or laparoscopy should be considered. A systematic review of the data has shown a similar outcome for both approaches. Metwally et al.36 suggest that there is no statistically significant difference in live birth rate, pregnancy rate, miscarriage rate, and preterm delivery rate between open and laparoscopic myomectomy.

Yet, for the laparoscopic procedure, reduced blood loss and shorter inpatient stays were noted.17 In laparoscopic myomectomies, a significantly lower risk of infertility fostering adhesions were found in comparison to laparotomy.16 Another general risk includes uterine rupture during pregnancy or labor, leading to a higher rate of caesarean section, of which is preventable. However, it is unclear if laparoscopic myomectomy increases the risk of rupture significantly. 16 Kameda et al.37 found that laparoscopic uterine myomectomy was successful in $45.7 \%$ of infertile patients with no known cause but fibroids in comparison to $28.6 \%$ without laparoscopic treatment, yet the difference was not significant. If the number of fibroids was $\leq 3$, the difference became statistically significant. Also, in patients with four-nine fibroids, the myomectomy showed a pregnancy rate of $64.7 \%$ with no pregnancies in patients without myomectomy. In patients with $>10$ fibroids however, no pregnancy could be achieved.

\section{Medical Treatment:}

While the application of hormones, through, for instance, the combined oral contraceptive pill, progesterone-only pill, and levonorgestrel intrauterine systems, is commonly used to improve pain and menstrual complaints dueto fibroids, they are not applicable to infertile women because of their contraceptive effect. Ulipristal acetate (UPA), a progesterone receptor modulator, is a licensed option for uterine fibroids because of its size reducing effect by increasing the apoptosis of leiomyoma cells. The therapy is limited to 3 months and its effect lasts for about 6 months without surgery. Luyckx et al.38 conducted a study with 52 patients receiving UPA with 21 patients wishing to conceive. From 3 months after the end of UPA therapy, 15 of the 21 patients $(71.0 \%)$ became pregnant, with a total of 18 pregnancies, of which, 12 pregnancies resulted in the delivery of 13 babies and 6 resulted in miscarriages. Purohit et al.17 pointed out that the study done by Luyckx et al.38 had shown that despite the size reduction of fibroids and possible conception after the end of the UPA therapy, there has been a higher rate of miscarriages if women did not undergo myomectomy as well, thus, surgical management is additionally being recommended.

\section{Case scenario:}

37 years old para $1+0$, has NVD of full term male child 14 years old, from previous marriage, now newly married since 3 years trying to conceive, presented to emergency with sever lower abdominal pain and low grade fever since 3 days, with well-known bilateral PCOS, posterior wall fibroid, type 2 DM on metformin 500mg po od.

\section{On examination:}

Temp:38 orally, sever suprapubic tenderness, bulky tender uterus,VE: tender cervical motion, bilateral tender adnexa, speculumexamination, hyper trophic cervix with multiple infected nabothian cysts, bleeds on touch,vaginal swab and pap smear collected sent to cytology with FBC,CRP,Urine c\&s.

Patient admitted for triple antibiotic iv(dalacin c $900 \mathrm{mg}$ iv tid,gentamycin $80 \mathrm{mg}$ iv tid,metrolag $500 \mathrm{mg}$ iv tid), iv hydration,iv perfalgan iv $1 \mathrm{gm}$ prn, voltaren $100 \mathrm{mg}$ rectal supp PR bd.

MRI pelvis with contrast done, whichconfirm, bilateral pcos, posterior wall fibroid $4.5 \times 3.5 \mathrm{~cm}$, with quarry hydrosalpinx at left tube. Patient discharge after 2 days from hospital afebrile, nopain, nortenderness, on:

Doxycycline $100 \mathrm{mg}$ po bd $2 \mathrm{w}$

Metrolag 500mg po bd $2 \mathrm{w}$

Mefex $500 \mathrm{mg}$ pot id $2 \mathrm{w}$

Follow up visit in OPD,pap smear confirm sever inflammatory reaction, patient offered cryocautary of cervix, Zithromax $1 \mathrm{gm}$ po stat

BMI was $32 \mathrm{kgm} / \mathrm{m} 2$,Ptient advised: 
Weight reduction, exercise, life style modification, dietian referral for control of type $2 \mathrm{DM}$, and pcos, metformin $500 \mathrm{mg}$ xr bd

Semen analysis of husband repeated, was normal.

Patient and her husband councelled abour laparoscopy for, myomectomy, pelvic adhesolysis,and excision of left hydrosalpinx if confirmedintraoperative, tubal patency test, both partners agreed for the plane.

Laparoscopydone, posterior wall myomectomy done, left tube was not dilated, it was fixed to posterior uterine wall by fibrous adhesions with signs of sever inflammatory reaction, looks like prohepted pelvic abscess? Adhesolysis of peritubal adhesions with release of whole left tube, adhesolysis of other pelvic adhesions, pelvic wash and irrigation with normal saline, tubal patency test with methylene blue injection Trans cervical was negative, no peritoneal spill intraoperative.

Patient kept in hospital 2 days post-operative on triple antibiotic again (dalacin c $900 \mathrm{mg}$ iv tid, gentamycin $80 \mathrm{mg}$ iv tid, metrolag 500mg iv tid) discharged home on:

1. Doxycycline $100 \mathrm{mg}$ po bd $2 \mathrm{w}$

2. Metrolag $500 \mathrm{mg}$ po bd $2 \mathrm{w}$

3. Reparil tab pot id $2 \mathrm{w}$

4. Metformin 500mg XR po bd

5. Weight reduction, lifestyle modification

Referral to IVF center for further management at least 6 months post-operative.

Patient attend the OPD clinic 7 weeks post-operative with BMI: $28 \mathrm{kgm} / \mathrm{m} 2$, amenorrhea 7 weeks, BHCG was positive in blood equal to 5-6 weeks pregnancy. Pelvic uss confirm IUGS 6weeks,positive FHR.,at 12 weeks NT done was normal,cervix was short $2.6 \mathrm{~cm}$ and funnling,patient offered cervical cerclage,glycemic control continued by diet control and metformin $500 \mathrm{mg}$ xr bd,pregnancy continued till $38 \mathrm{weeks}+5$ days ,cervical cerclage removed at 37 weeks, elective lscs at $38 \mathrm{w}+5 \mathrm{~d}$ after steroid coverage. Full term living male baby $3.5 \mathrm{kgm}$ with good apgar scor,both patient and baby went home 2 days after lscs.

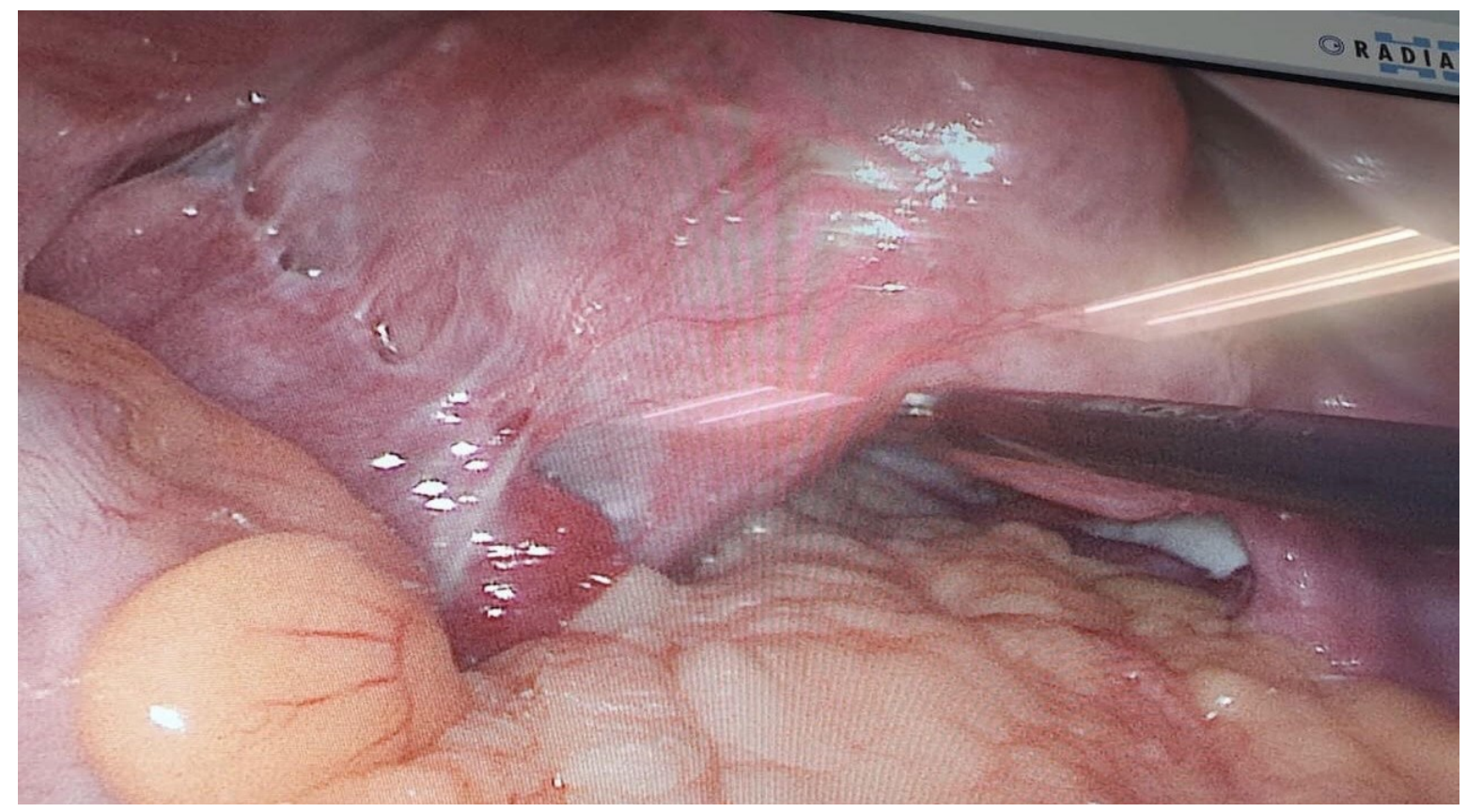

Left tube Adherent to posterior Uterine wall with Myoma 


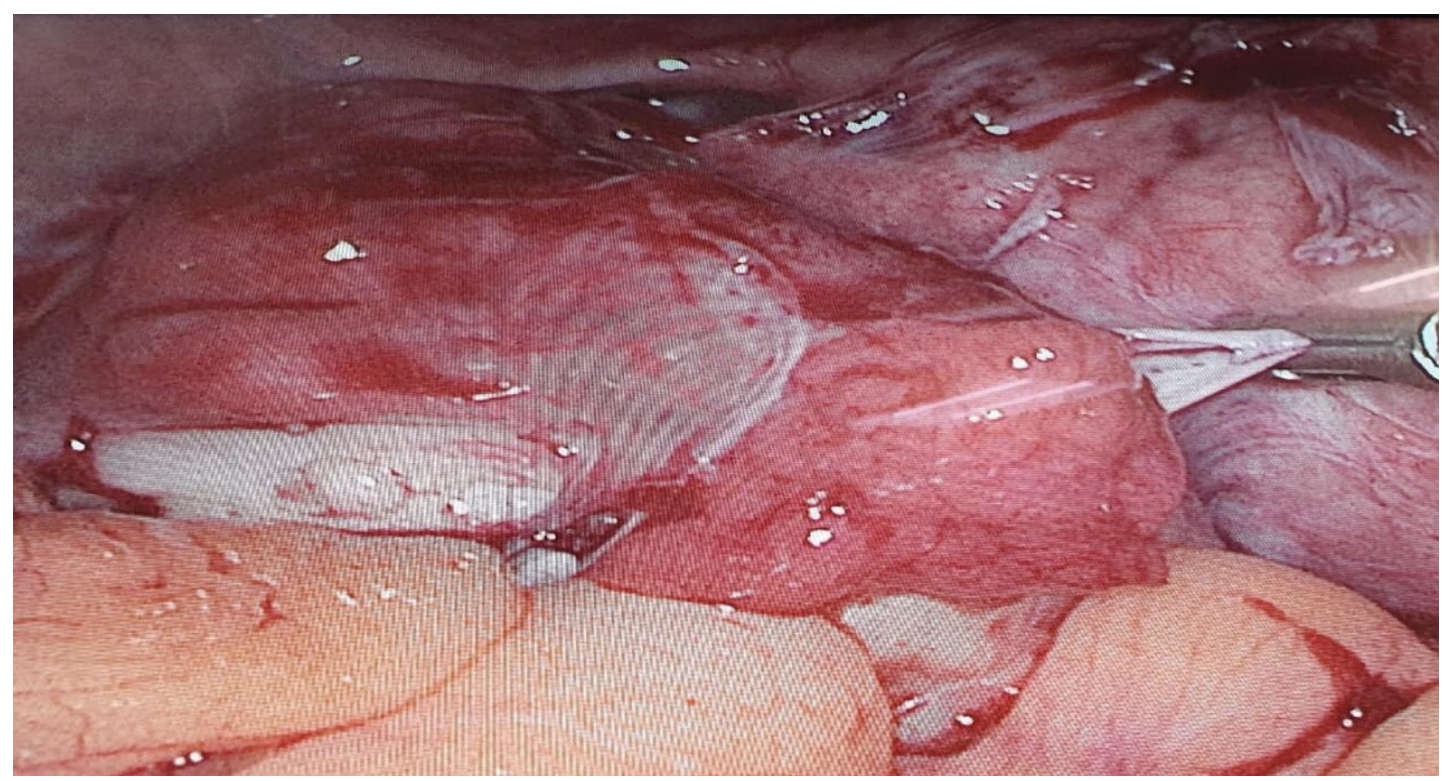

Left tube after Adhesolysis.

\section{Discussion:-}

\section{Diagnosis and management of PID:}

All women with suspected PID should have a pelvic examination to evaluate for cervical discharge, cervical motion tenderness, uterine tenderness, adnexal tenderness or masses. The diagnosis of pelvic inflammatory disease is clinical and is defined by lower genital tract inflammation such as cervical discharge, an increased number of white blood cells on wet prep, or cervical friability.

As stated before, the diagnosis of pelvic inflammatory disease is primarily clinical. PID should be considered in any sexually active young woman with pelvic or low abdominal pain and evidence of genital tract tenderness on exam. While laboratory tests may help confirm the diagnosis, NAAT testing typically can take several hours to days to result depending on your institution. Negative results do not exclude the diagnosis. An ultrasound or CT without findings of PID does not exclude the diagnosis. Therefore, early and prompt treatment should be started based on clinical suspicion.[10][11][1 Empiric treatment for PID in the inpatient setting includes:

1. Cefotetan (2 g intravenously [IV] every 12 hours) plus doxycycline (100 $\mathrm{mg}$ by mouth every 12 hours) or

2. Cefoxitin ( $2 \mathrm{~g}$ IV every 6 hours) plus doxycycline ( $100 \mathrm{mg}$ by mouth every 12 hours) or

3. Clindamycin ( $900 \mathrm{mg}$ IV every 8 hours) plus gentamicin ( 3 to $5 \mathrm{mg} / \mathrm{kg}$ IV once daily)

The CDC recommends the following for first-line treatment for outpatient therapy:

1. Doxycycline ( $100 \mathrm{mg}$ orally twice a day for 2 weeks) plus ceftriaxone $250 \mathrm{mg}$ intramuscularly (IM) for one dose $\boldsymbol{o r}$ cefoxitin $2 \mathrm{~g}$ IM with probenecid (1g orally) for one dose $\boldsymbol{o r}$ another parenteral third-generation cephalosporin

Metronidazole (500 mg orally twice per day for 14 days) should be added if there is a concern for trichomonas or recent vaginal instrumentation.

2] Delayed treatment of PID has a strong association with worsened outcomes and long-term complications. However, even with timely treatment, long-term complications can occur

Chronic pelvic pain is seen in as many as one-third of women with PID. The pain is thought to be related to inflammation, scarring, and adhesions from the infectious process. The strongest predictor of developing chronic pelvic pain related to PID is recurrent PID.

Infertility can also result from PID, regardless if the patient is symptomatic or asymptomatic from the pelvic infection.

The infection can cause severe damage to the fertility can be pronounced fallopian tubes, including loss of the ciliary epithelial cells of the fallopian tube and occlusion of the tube. 
The effects of fibroids on fertility and its management on improving fertility are inconclusive. The management should be individualized depending on the classification of the fibroids in relation to endometrium. Hysteroscopic excision of the submucosal fibroids should be considered before starting fertility treatment. With regards to intramural fibroids, it is individualized according to its relation to the uterine cavity. The subserosal fibroid is unlikely to have an impact on fertility. Medical, UPA, and MrgRUS are not recommended for women who desire fertility treatment.

\section{Recommendations:-}

Infertility cases has to be managed systemically

First:

Early intervention with iv antibiotics, till fever and pain subside, followed by oral doxycycline and metrolag po bd $2 \mathrm{w}$. to prevent further complications of PID.

\section{Second:}

Management of chronic cervicitis as it work as septic focus.

\section{Third:}

Good management of PCOS, with weight reduction, life style modifications, correct dose of metformin 1-2.5 gm daily.

\section{Fourth:}

Surgical myomectomy is the best option for infertility

Fifth:

Laparoscopic adhesolysis with myomectomy and pelvic wash and irrigation, has golden role in infertility management.

\section{Six:}

Good preparation of patient before referral to IVF might help her to conceive naturally, or even give good result with less cycles of IVF, and get baby home.

\section{References:-}

1. Woodhall SC, Gorwitz RJ, Migchelsen SJ, Gottlieb SL, Horner PJ, Geisler WM, Winstanley C, Hufnagel K, Waterboer T, Martin DL, Huston WM, Gaydos CA, Deal C, Unemo M, Dunbar JK, Bernstein K. Advancing the public health applications of Chlamydia trachomatis serology. Lancet Infect Dis. 2018 Dec; 18(12):e399-e407.

2. Basit H, Pop A, Malik A, Sharma S. StatPearls [Internet]. StatPearls Publishing; Treasure Island (FL): May 24, 2020. Fitz-Hugh-Curtis Syndrome.

3. Stevens JS, Criss AK. Pathogenesis of Neisseria gonorrhoeae in the female reproductive tract: neutrophilic host response, sustained infection, and clinical sequelae. Curr. Opin. Hematol. 2018 Jan;25(1):13-21

4. Molenaar MC, Singer M, Ouburg S. The two-sided role of the vaginal microbiome in Chlamydia trachomatis and Mycoplasma genitalium pathogenesis. J. Reprod. Immunol. 2018 Nov;130:11-175.

5. Di Tucci C, Di Mascio D, Schiavi MC, Perniola G, Muzii L, Benedetti Panici P. Pelvic Inflammatory Disease: Possible Catches and Correct Management in Young Women. Case Rep Obstet Gynecol. 2018;2018:5831029.

6. Risser WL, Risser JM, Risser AL. Current perspectives in the USA on the diagnosis and treatment of pelvic inflammatory disease in adolescents. Adolesc Health Med Ther. 2017;8:87-94

7. Ross J, Guaschino S, Cusini M, Jensen J. 2017 European guideline for the management of pelvic inflammatory disease. Int J STD AIDS. 2018 Feb;29(2):108-114.

8. .Wang Y, Zhang Y, Zhang Q, Chen H, Feng Y. Characterization of pelvic and cervical microbiotas from patients with pelvic inflammatory disease. J. Med. Microbiol. 2018 Oct;67(10):1519-1526.

9. Jin BB, Gong YZ, Ma Y, He ZH. Gynecological emergency ultrasound in daytime and at night: differences that cannot be ignored. Ther Clin Risk Manag. 2018;14:1141-1147

10. Jensen JS, Cusini M, Gomberg M, Moi H. Background review for the 2016 European guideline on Mycoplasma genitalium infections. J Eur Acad Dermatol Venereol. 2016 Oct; 30(10):1686-1693. 
11. Das BB, Ronda J, Trent M. Pelvic inflammatory disease: improving awareness, prevention, and treatment. Infect Drug Resist. 2016; 9:191-7.

12. Brun JL, Graesslin O, Fauconnier A, Verdon R, Agostini A, Bourret A, Derniaux E, Garbin O, Huchon C, Lamy C, Quentin R, Judlin P., Collège National des Gynécologues Obstétriciens Français. Updated French guidelines for diagnosis and management of pelvic inflammatory disease. Int J Gynaecol Obstet. 2016 Aug; 134(2):121-5.

13. Colombel JF, Shin A, Gibson PR. AGA Clinical Practice Update on Functional Gastrointestinal Symptoms in Patients With Inflammatory Bowel Disease: Expert Review. Clin. Gastroenterol. Hepatol. 2019 Feb;17(3):380390.e1

14. Witkin SS, Minis E, Athanasiou A, Leizer J, Linhares IM. Chlamydia trachomatis: the Persistent Pathogen. Clin. Vaccine Immunol. 2017 Oct; 24(10) .

15. McVeigh E et al. Oxford University Press, Oxford Handbook of Reproductive Medicine and Family Planning (2008), Oxford, UK.

16. Desai P, Patel P. Fibroids, infertility and laparoscopic myomectomy. J Gynecol Endosc Surg. 2011;2(1):36-42.

17. Purohit P, Vigneswaran K. Fibroids and infertility. Curr Obstet GynecolRep. 2016; 5:81-88.

18. Ciavattini A et al. Uterine fibroids: Pathogenesis and interaction with endometrium and endomyometrial junction. Obstet Gynecol Int. 2013; 2013:173184.

19. Yoshino $\mathrm{O}$ et al. Decreased pregnancy rate is linked to abnormal uterine peristalsis caused by intramural fibroids. Hum Reprod. 2010; 25(10):2575-9.

20. Voronin DN et al. [Relationship between activation of endometrial CD56+ natural killers and the character of leiomyoma nodules growth in patients with uterine leiomyoma]. Klein Lab Diagn. 2018; 63(2):119-23. (In Russian).

21. Pritts EA et al. Fibroids and infertility: An updated systematic review of the evidence. Fertil Steril. 2009; 91(4):1215-23.

22. Tinelli A et al. Sub mucous fibroids, fertility, and possible correlation to pseudo capsule thickness in reproductive surgery. Biomed Res Int. 2018; 2804830:1-7.

23. Vam Heertum K, Barmat L. Uterine fibroids associated with infertility. Women's Health (Lond). 2014; $10(6): 645-53$.

24. Wang $\mathrm{X}$ et al. The impact of concavity-distorting intramural fibroids on the efficacy of in vitro fertilizationembryo transfer: An updated meta-analysis. BioMed Res Int. 2018; 8924703:1-13.

25. Rackow BW, Taylor HS. Submucosal uterine leiomyomas have a global effect on molecular determinants of endometrial receptivity. Fertil Steril. 2010; 93(6):2027-34.

26. Sinclair DC et al. Leiomyoma simultaneously impair endometrial BMP-2-mediated decasualization and anticoagulant expression through secretion of TFG-33. J Clin Endocrinal Metab. 2011; 96(2):412-21.

27. Pritts EA. Fibroids and infertility: A systematic review of the evidence. Obstet Gynecol Surv. 2001; 56(8):48391.

28. Zepiridis LI et al. Infertility and uterine fibroids. Best Pract Res Clin Obstet Gynaecol. 2016; 34:66-73.

29. Casini ML et al. Effects of the position of fibroids on fertility. Gynecol Endocrinol. 2006; 22(2):106-9.

30. Bulletti C et al. The role of leiomyomas in infertility. J Am Assoc Gynecol Laparosc. 1999;6(4):441-5

31. Lisiecki $M$ et al. Fertility impairment associated with uterine fibroids - a review of literature. Prz Menopauzalny. 2017; 16(4):137-40.

32. Vimercati A et al. Do uterine fibroids affect IVF outcomes? Reprod Biomed Online. 2007; 15(6):686-91.

33. Klatsky PC et al. The effect of fibroids without cavity involvement on ART outcomes independent of ovarian age. Hum Reprod. 2007; 22(2):521-6.

34. Somigliana E et al. Fibroids not encroaching the endometrial cavity and IVF success rate: A prospective study. Hum Reprod. 2011; 26(4):834-9.

35. Sunkara SK et al. The effect of intramural fibroids without uterine cavity involvement on the outcome of IVF treatment: A systematic review and meta-analysis. Hum Reprod. 2010; 25(2):418-29.

36. Metwally $M$ et al. Surgical treatment of fibroids for subfertility. Cochrane Database Syst Rev. 2012; 11:CD003857.

37. Kameda S et al. Utility of laparoscopic uterine myomectomy as a treatment for infertility with no obvious cause except for uterine fibroids. Gynecol Minim Invasive Ther. 2018; 7(4):152-5.

38. Luyckx $M$ et al. First series of 18 pregnancies after ulipristal acetate treatment for uterine fibroids. Fertil Steril. 2014; 102(5):1404-9. 